\title{
Editorial: Challenges and Strategies in Plant Biology Research
}

\author{
Ashwani Pareek ${ }^{1} \cdot$ Viswanathan Chinnusamy $^{2}$
}

Published online: 24 November 2016

(C) Indian Society for Plant Physiology 2016

Global agricultural productivity must be increased significantly to meet food, feed and fuel requirements of growing population. To achieve this, plant biologists must unlock the rules of life to predict genotype-phenotype relationship in diverse environments and pyramid these traits for enhancing agricultural productivity and environmental sustainability. Plant biology is currently passing through a very exciting phase where technological innovations and high throughput analytical platforms are lending an unprecedented support towards generation of new knowledge. Nonetheless, new challenges to plant productivity are also being perceived, thereby making it essential to strategize the plant research in new avenues. In this endeavour, plant physiology remains the key area of study, as it addresses fundamental questions about adaptations in plants, enabling them to survive under the changing climatic conditions. The theme of the 3rd International Plant Physiology Congress (IPPC-2015) was chosen to be the 'Challenges and Strategies in Plant Biology Research', which was held at Jawaharlal Nehru University (JNU) during December 11-14, 2015. The meeting was co-hosted by The Indian Society of Plant Physiology (ISPP), National Institute of Plant Genome Research (NIPGR) and JNU. The American Society of Plant Biology (ASPB) was also an active partner during this meeting. This special issue is being published in commemoration of this historical event

Ashwani Pareek

ashwanip@mail.jnu.ac.in

1 Stress Physiology and Molecular Biology Laboratory, School of Life Sciences, Jawaharlal Nehru University, New Delhi, India

2 Division of Plant Physiology, Indian Agricultural Research Institute, New Delhi, India where 18 detailed proceedings from leading talks have been compiled and is being made available to the readers. The manuscripts presented in this issue are both review and original research articles.

Use of OMICs technologies is the key for trait discovery. Four review articles in this issue summarize the advances and prospects of transcriptomics (The use of NGS technology in deciphering transcriptome of medicinal plants), metabolomics (Measuring The Plant Terpenome: Predicted vs. Actual Emission Potential) and phenomics (Phenomics: unlocking the hidden genetic variation for breaking the barriers in yield and stress tolerance and Plant phenotyping: A perspective) for gene and trait discovery. The article on "CRISPR-Cas9 and CRISPR-CPfl mediated tranegene free genome editing in rice" introduces the readers with precise way of functional validation of genes identified through various OMICs and specific editing of genomes for crop improvement, specifically rice.

Abiotic stresses such as extremes of temperature (low and high) and water (both drought and flood), poor quality of water, pose a major threat to agriculture. Global climate change further exacerbates these stresses. Keeping in mind the importance of this research, nine articles in this special issue illustrate the current research trends in biotic and abiotic stress tolerance of plants. Two articles (Rice and heat stress biology, and Phenotyping for improving high temperature stress tolerance in rice) have highlighted the latest research in the area of high temperature stress. Similarly two articles (Reproductive Stage Salinity Tolerance in Rice: A complex trait to phenotype, and Physiological characterization of gamma-ray induced mutant population of rice to facilitate biomass and yield improvement under salinity stress) deals with standard phenotyping protocol and identification of novel sources 
for improving salinity stress tolerance in rice. The article on "Physiological characterization and grain yield stability analysis of Recombinant Inbred Lines under drought stress in Wheat" talks about the physiological approaches to improve grain yield and stability of this major food crop under drought conditions. The role of methyl glyoxal which is central to many abiotic stresses is also being discussed in a review on "Methylglyoxal detoxification in plants: role of glyoxalase pathway".

Next to water, nitrogen is the major driver of crop productivity. However, currently nitrogen use efficiency is very low. One article discusses the "Advances in genetic basis of nitrogen use efficiency of rice". An article on "Coconut (Cocus nucifera) response to climate change variables and adaptation strategies" has been included to introduce the readers with the physiological adaptations in perennial crops. Abiotic stresses such as drought is expected to increase the loss caused by biotic stresses such as nematodes in major food crops. An article on "Molecular aspects of plant-nematode interaction" was included in this issue to cover the area of biotic stress.

Grain quality is an important determinant for human nutrition. Rice is a major staple food of the world, and modification of rice starch quality is important for cooking quality and low glycemic index. The article on "Sequence polymorphism in the Waxy locus and its relationship with apparent amylose content of endosperm starch in cultivars of rice (Oryza sativa L.) from Northeast India" illustrates the natural variation for amylase/amylopectin content and means to alter this ratio in rice for improving quality.

In addition to the above areas, three articles deals with various basic aspects of plant development, such as root development (A review of genetic and molecular mechanisms that control post-embryonic root radial patterning), fruit set (Floral biology and biochemical changes in stigma and pollen in mango cultivars differing in fruit set) and senescence (How and why of flower senescence-Understanding from models to ornamentals).

Editors are thankful to all the authors who have contributed these interesting articles and the reviewers who have lend us constant support for reviewing these manuscripts on time. Editors hope that this issue of Indian Journal of Plant physiology will find a good use in scientific research and will contribute significantly in designing future strategies to handle the challenges of climate change, through plant Physiology. 